JOINT COMMISSION VI

Solar \& Stellar Coronae

Commissions $10 / 12$ with $36 \& 44$ with

Chairman \& Editor: R. Pallavacini 


\title{
HEATING AND MOMENTUM DEPOSITION IN HOT STARS
}

\author{
J. I. CASTOR \\ Lawrence Livermore National Laboratory \\ Box 808, Livermore, CA 94550, U.S.A.
}

The one great point of similarity between the coronae of hot and cool stars is that both are spatially extended regions of more-or-less tenuous gas that is flowing outward, and at least some of which is at a temperature in excess of $10^{6} \mathrm{~K}$. Coronae defined in this way are almost universal among stars-excepting cool supergiants-but this similarity may hide significant differences in the processes that produce coronae. There are two rather different paradigms for their origin: the cool-star paradigm and the hot star paradigm.

The coronae of cool stars like the sun are of such a low density that radiative cooling is inefficient; the outward flow is weak enough that there is a fairly extended subsonic flow region. The outer corona is heated by a flux of wave energy, and some of this energy is then conducted inward to heat the inner corona. The outflow is driven by the pressure of the heated gas, assisted perhaps by wave pressure. The structure of the corona is greatly affected by the topology of the magnetic field, which channels the outflow and governs the transport of energy and momentum by waves.

The hot star coronae have rather different properties. In the O-type stars and $\mathrm{B}$ supergiants the corona is so dense that any material raised to temperatures above $10^{6} \mathrm{~K}$ quickly cools to radiative equilibrium. Thus energy deposition is not a significant issue in the coronae of hot stars. (These considerations may not apply for B stars near the main sequence, where the major interest is in the Be phenomenon.) Observations suggest a. rather quickly accelerated wind in hot stars, so a large part of the corona is expanding supersonically. Radiation pressure in many spectral lines is thought to provide the momentum to drive the wind. The coronae fluctuate in time, and have significant spatial in homogeneities, but the origin of these is uncertain.

What is known about the morphology of hot star coronae is, in summary: at the crudest level a steady spherical outflow close to radiative equilibrium, indicated by $P$ Cygni profiles of UV resonance lines and the radio spectrum; superimposed on this steady flow are fluctuations of density and velocity in times of a few hours; some hot $\left(\right.$ few $\left.\times 10^{6} \mathrm{~K}\right)$ gas is interspersed with the cool material, and this emits $\mathrm{x}$-rays which in turn produce high-ionization species like O VI; some stars have non-thermal radio spectra indicative perhaps of electron acceleration in shocks; some of the fluctuation phenomena have the rotation time scale. The size and shape of the structures associated with the fluctuations are unknown, apart from limits that can be set based on the visibility of the fluctuations.

The major source of momentum for the hot star wind is line absorbtion or scattering. Other sources of momentum for the wind might be sound waves and Alfvén waves; these suffer the objection that for a significant effect in OB stars the wave amplitudes would be excessive. The theory of the radiation-driven wind was developed by Lucy and Solomon (1970), Castor, et al. (1975), Abbott (1982), Friend and Abbott (1986), and 
Pauldrach, et al. (1986), among others. The Sobolev line transfer approximation for making steady wind models has been substantiated. However, the Sobolev approximation fails in the presence of small-scale velocity fluctuations, and a more accurate theory reveals a strong radiation-driven hydrodynamic instability ( $c f$. ., MacGregor, Hartmann and Raymond 1979; Owocki and Rybicki 1984, 1985). This produces of order 100 linear e-folds of amplification as a perturbation is carried out through the wind! Numerical simulations with various simplifications are so far the only guide to the fully developed form of the instability (Owocki et al. 1988, Castor 1991, Owocki 1991). These have shown either large amplitude self-excited oscillations or strong amplification of waves applied at the photosphere, depending on stellar parameters such as line width/sound speed, and also on the details of the radiative transfer. The structures in either case consist of large-scale rarefaction waves terminated by reverse shocks and accompanied in some cases by forward shocks. These structures resemble those needed to explain the production of $x$-rays and the narrow absorption components in UV P Cygni lines.

Two important aspects of hot star coronal morphology are the common non-radial pulsations (NRP) and the narrow absorption components (NAC). The NRP are inferred from drifting absorption dips in rotationally broadened photospheric absorption lines. These observations are consistent with $\ell \approx-m=2-10$ and periods of a few hours. The amplitudes are of order $10-20 \mathrm{~km} \mathrm{~s}^{-1}$. The NAC appear as blueward-accelerated dips in the UV resonance lines. The acceleration lasts of order half a day, following which the absorption fades out in one to a few days. These time scales seem to vary in proportion to the rotation period (Prinja 1988).

The NAC are the major clue to the hot star coronal morphology, and no fully satisfactory theory exists at present. A severe difficulty with models like that of Owocki, et al., is that the apparent acceleration of the NAC is several times less than the typical wind acceleration; the coupling to stellar rotation is another difficulty. The kind of model suggested by the observations is one with dense plumes anchored to the photosphere (or the NR.P wavefronts), as in Mullan's (1984) co-rotating interaction region picture. The quantitative aspects remain to be developed.

This work was performed under the auspices of the U. S. Department of Energy by the Lawrence Livermore National Laboratory under Contract No. W-7405-ENG-8.

\section{References}

Abbott, D.C.: 1982, Astrophys. I., 259, 282.

Castor, J.I.: 1991, in Stellar Atmospheres: Beyond Classical Models (L. Crivellari, I.

Hubeny and D. G. Hummer, eds.), Kluwer, Dordrecht, p. 221.

Castor, J.I., Abbott, D.C. and Klein, R.I.: 1975, Astrophys. .J., 195, 157.

Friend, D.B. and Abbott, D.C.: 1986, Astrophys. I., 311, 701.

Lucy, L.B. and Solomon, P.: 1970, Astrophys. J., 159, 879.

MacGregor, K.B., Hartmann, L. and Raymond, J.: 1979, Astrophys. .J., 231, 514.

Mullan, D.J.: Astrophys. J., 283, 303.

Owocki, S.P.: 1991, in Stellar Atmospheres: Beyond Classical Models (L. Crivellari, I.

Hubeny and D. G. Hummer, eds.), Kluwer, Dordrecht, p. 235.

Owocki, S.P., Castor, J.I. and Rybicki, G.B.: 1988, Astrophys. .J., 335, 914.

Owocki, S.P. and Rybicki, G.B.: 1984, Astrophys. .J., 284, 337.

Owocki, S.P. and Rybicki, G.B.: 1985, Astrophys. .J., 299, 265.

Pauldrach, A., Puls, J. and Kudritzki, R.P.: 1986, Astron. Astrophys., 164, 86.

Prinja, R.K.: 1988, Monthly Not. Roy. Astr. Soc., 231, 21P. 